\section{New Capabilities for the TEM: Automatic Orientation Measurement and Nanocrystal Grain Maps}

S.I. Wright, D.J. Dingley and P.R. Mainwaring TSL, Inc. 392 East 12300 South, Draper, Utah 84020

\section{Introduction}

Orientation Imaging Microscopy (OIM) is a rapid and spatially specific technique for automatically measuring individual crystallographic orientations in a polycrystalline sample $e^{1,2}$. The technique is based on electron backscatter diffraction in the scanning electron microscope (SEM). While the OIM technique has seen many applications to the investigation of structure/ property relationships in polycrystalline materials, with grain sizes ranging from millimeters to submicron, it is not easily applied to the characterization of microstructures at the nanometer scale due to the inherent resolution limitations of the SEM. Thus, a complementary technique for the transmission electron microscope (TEM) would be advantageous for the study of local orientation in submicron structures such as those that exist in nanocrystalline materials and deformed materials. The approach most equivalent to OIM in the SEM would be to move the beam on a prescribed grid and collect Kikuchi diffraction or convergent beam patterns at each point. The patterns could be indexed and the orientation determined at each point. However, such an approach is impractical on the TEM because neither Kikuchi patterns nor convergent beam patterns can be obtained reliable at the $10 \mathrm{~nm}$ grain size level.. The proposed method for adapting the OIM technique to the TEM is to use dark field imaging coupled with computer control of the electron beam. This article describes a new technique (U.S. patent $5,576,543$ and others) for automated spot pattern indexing and TEM-based orientation imaging.

\section{Basic Diffraction in the TEM}

A diffraction pattern consisting of bright spots (see Figure 1b) is obtained when a single crystal sample is viewed in a TEM using parallel illumination as shown in the schematic in Figure 1a.
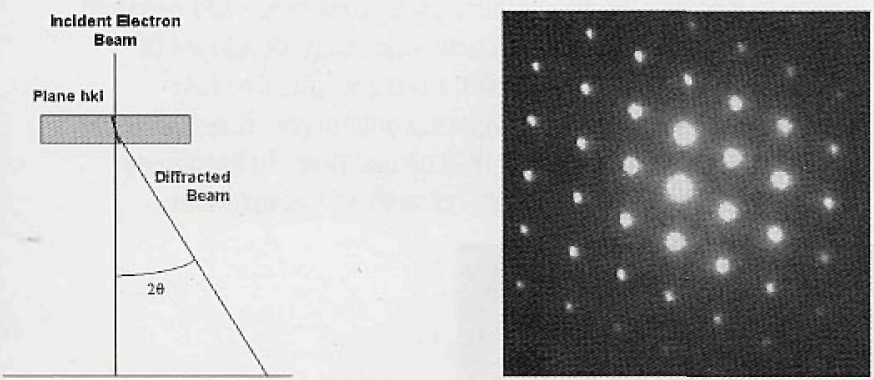

Figure 1 - (a) Schematic of diffraction in the TEM and (b) a spot pattern for $a<111>$ oriented silicon single crystal.

If the crystal is aligned in a crystallographically symmetric orientation with respect to the incident beam, these spots will form a symmetric pattern as shown in Figure 1b. In other orientations, the crystal will produce an array of spots that is not as symmetric.

The diffraction condition is governed by the Bragg equation,

$$
\lambda=2 d_{h k l} \sin \theta
$$

for a given plane denoted by the Miller indices $h k l . \lambda$ is the wavelength of the incident beam, $d_{h k l}$ is the lattice spacing and $\theta$ is the angle of incidence of the electron beam with the dif- fracting plane. The orientation of the diffracting crystal can be determined by the geometrical arrangement of spots in the pattern. However, when a polycrystalline sample is viewed, the incident electron beam is generally sufficiently large to cause simultaneous diffraction from multiple grains. The resulting diffraction typically appears as shown in Figure 2.

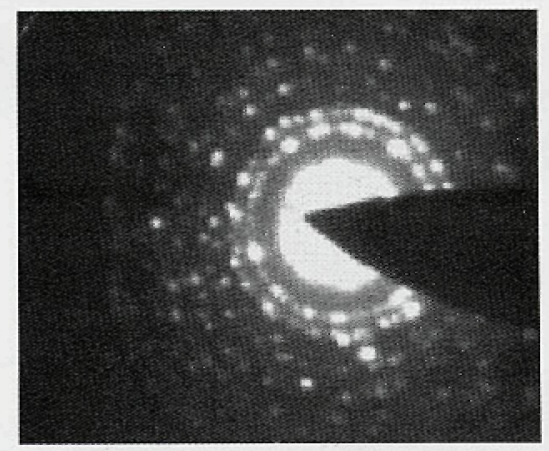

Figure 2 - Ring diffraction pattern for nanocrystalline aluminum.

A polycrystalline sample with a random distribution of grain orientations would produce a pattern consisting of a set of concentric rings. The ring pattern is essentially a superposition of the individual spot patterns of the diffracting grains. It is not possible, by inspection of the ring pattern alone, to distinguish which spots originate from which crystals in the specimen. However, the orientation of the individual grains contributing their individual spot patterns to the ring pattern can be determined using successive dark field images.

\section{Dark Field Scanning, Hollow Cone and Conical Scanning Microscopy}

The incident angle of the electron beam can be altered to force part of a diffraction ring to pass through the objective aperture to form a dark field image as shown in Figure 3. Only those crystals or parts of crystals that are oriented such that one of their diffraction spots lies in the portion of the diffraction ring passing through the objective aperture will appear bright in the dark field image.

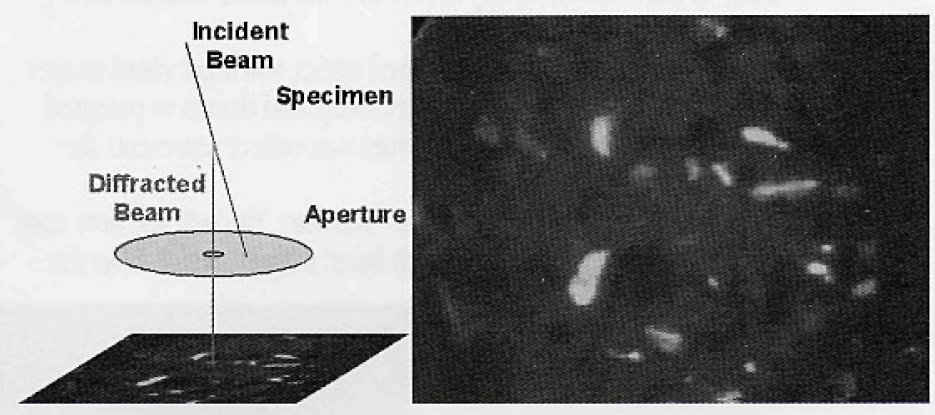

Figure 3 - (a) Schematic of dark field image formation and (b) a sample dark field image from an aluminum polycrystal.

If the beam is rotated to a new position along the diffraction ring (i.e., the tilt of the beam is maintained constant) then crystals which appeared bright in the previous dark field image at the former beam position will appear dark in the dark field image at the new beam position, while other crystals which were dark at the previous beam position will brighten up at the new position. An example of three successive dark field images for a beam rotating along a diffraction ring to three different positions is shown in Figure 4.

Continued on page 14 


\section{WINEDS \\ HIGH PERFORMANCE XRAY MICROANALYSIS}

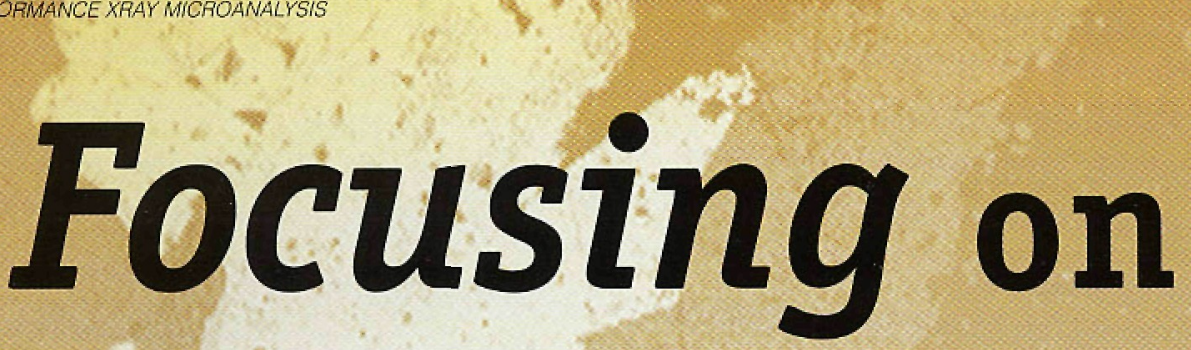

Simplicity and Value

This fast, accurate 32-bit PC Windows" based analyzer

works like your other Windows"' applications and it's a

much better value than any other microanalysis system

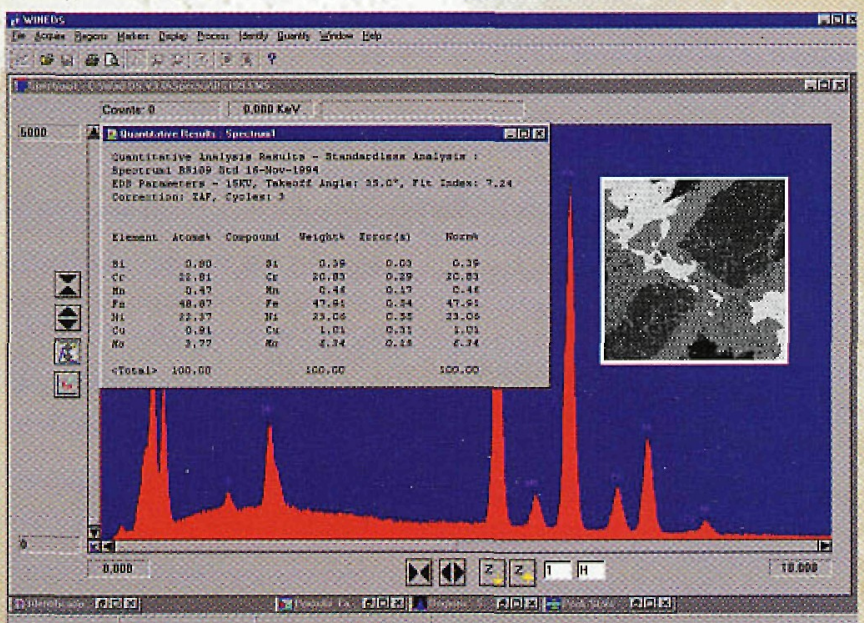

- New Quant Wizard one step analysis

- WINEDS PX9000 Pulse Processor DX3000 Bias

Interfaces to several detector brands

5 to $10 \mathrm{eV}$ improvement on most detectors resolutions

- Complete new systems - including new Gresham detectors

- Integrated active scan or passive digital imaging systems

- Partial upgrades to suit your system needs

TNAS pays top dollar for trade-ins, offers service for TN5500, TN2000 systems and repairs or upgrades existing $\mathrm{X}$-ray detectors
TN ANALYZER SERVICE INC. 7897 HWY 19 Dane,WI 53529 Phone 608.798.2005 Fax608.798.1675 email doug_tnas@msn.com Web WWW.tnanalyzerservice.com WWW.tnanalyzer.com

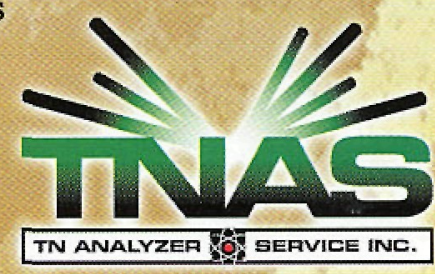


New Capabilities for the TEM: Automatic Orientation Measurement and Nanocrystal Grain Maps Continued from page 12
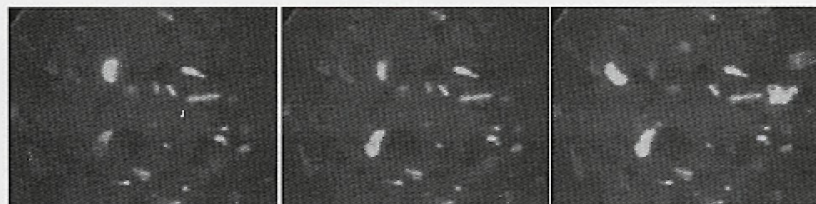

Figure 4 - Dark field images from three successive positions of the electron beam along a diffraction ring.

In practice, a video camera is positioned to enable successive dark field images to be digitized into a computer. The computer is interfaced to the TEM equipped with conical scan or hollow cone capability. This allows the computer to control the tilt $(\theta)$ and rotation $(p)$ of the electron beam. The computer tilts the beam to the first value of $\theta$ and then rotates the beam around the ring in two degree steps. At each step the resulting dark field image is captured by the computer and recorded. This procedure is repeated for up to 40 values of $\theta$. A schematic of the tilt and rotation parameters is shown in Figure 5

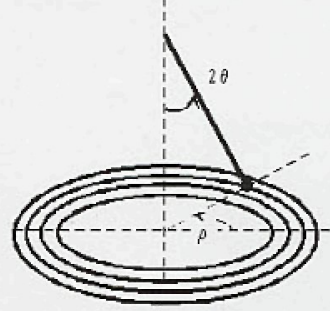

Figure 5 - Schematic showing the tilt (q) and rotate $(p)$ conical scan parameters.

\section{Orientation Determination}

Once this scanning procedure is completed, the intensity at every tilt and rotation step in the scan is known for every pixel in the dark field image field of view. This data can be used to reconstruct a spot pattern for any given point in the field of view. An example is shown in Figure 6 for a dark field scan on a sample of single crystal gallium arsenide. Figure $6 a$ shows a dark field image of the area scanned along with the pixel for which the "spot pattern" shown in $6 \mathrm{~b}$ is reconstructed. Figure $6 \mathrm{~b}$ clearly shows that there is some overlap in the bright up events between different rings and successive positions along the rings. This is due to the size of the objective aperture.
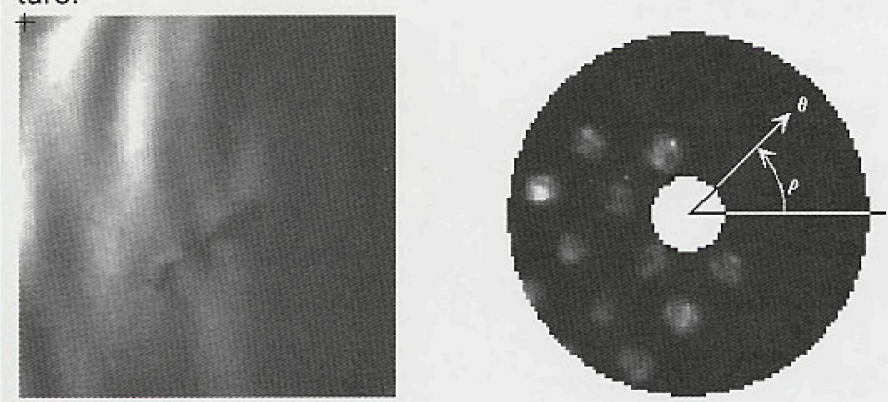

Figure 6 - (a) A dark field image and (b) a reconstructed spot pattern generated for the pixel denoted in (a) from the intensities recorded at successive rotations (j) and tilts $(\mathrm{q})$ of the incident electron beam.
Once a reconstructed spot pattern is formed, the orientation can be determined by indexing the spot pattern. An example of an automatically indexed, reconstructed spot pattern is shown in Figure 7 .

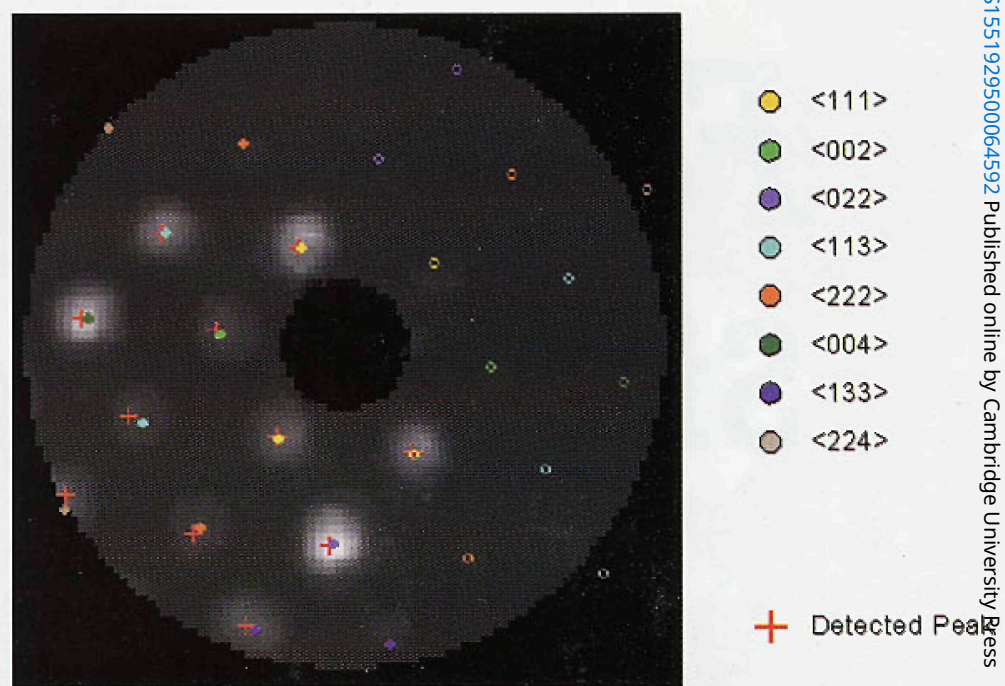

Figure 7 - Indexed reconstructed spot pattern.

\section{Grain Maps of Nanocrystalline Materials for}

Grain Size Analysis

Development and use of nanocrystalline materials for microelectronic applications is becoming increasingly important. In addition to grain orientation measurement in films, grain size is at least of equal importance. Armed with all the reconstructed spot patterns and the corresponding $X, Y$ coordinates of each pixel in the image, reconstructing a grain map is straightforward. Spot patterns are compared between successive pixels and when the orientation changes, a new grain is constructed. The resulting grain map is shown in Fig. 8. Each grain is colored a unique color to distinguish it from its neighbors. A grain size histogram is constructed from this grain map as shown in Fig. 9. Grain size statistics, such as area fraction and numbers of grains are also calculated for this distribution.

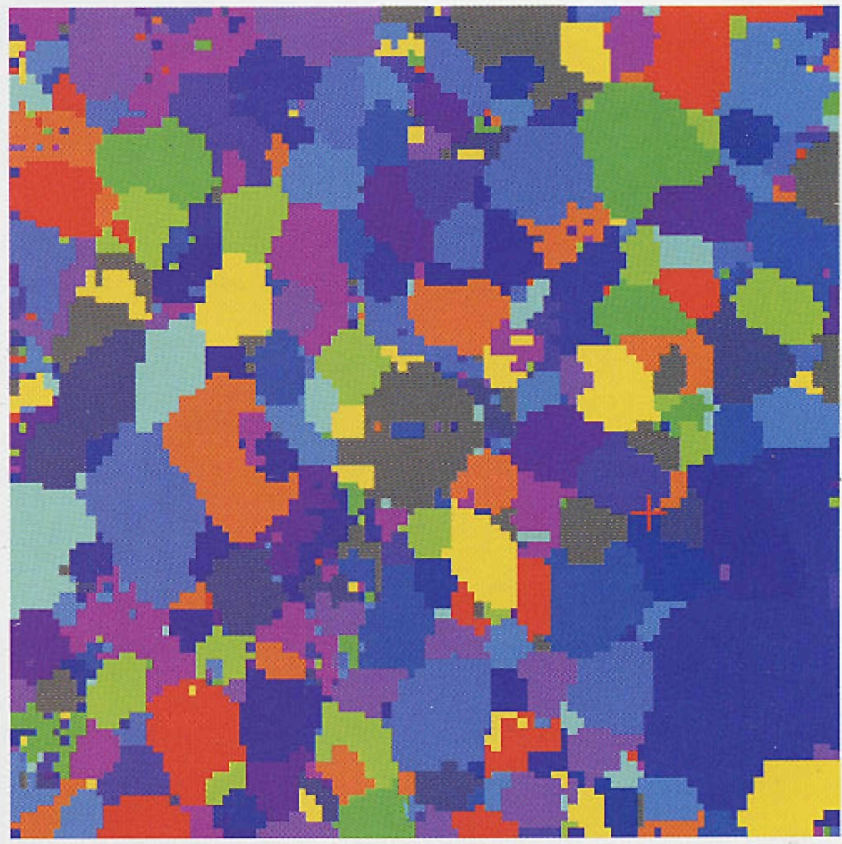

Figure 8 - Reconstructed Grain Map (Based on Spot Pattern Changes). 
Area Fraction

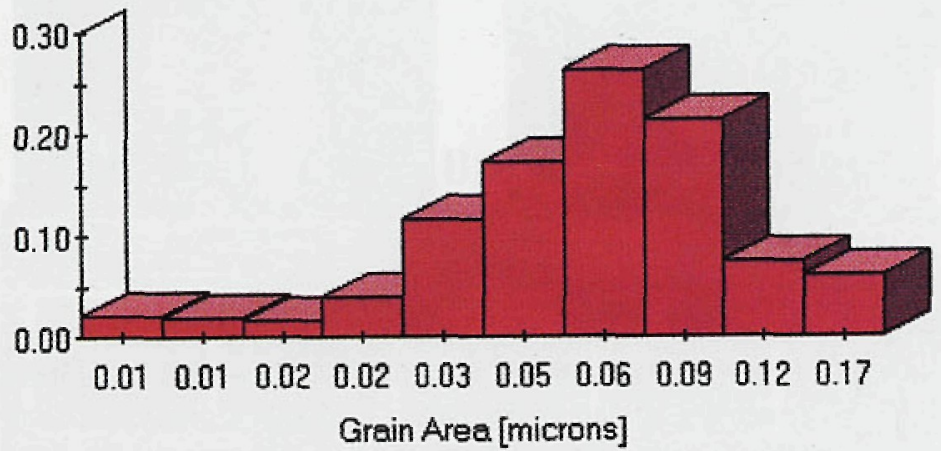

Figure 9 - Grain Area Histogram (Based on Figure 8)

References

[1] B. L. Adams, S. I. Wright, and K. Kunze, Met. Trans., 24A, (1993) p. 819.

[2] S. I. Wright, J. Computer Assisted Microscopy, 5, (1993) p. 207.

[3] T. C. Isabell and V. P. Dravid, Ultramicroscopy, (1997) in press

[4] M. H. Loretto, Electron Beam Analysis of Materials, 2 ed. (Chapman \& Hall, London, 1994).

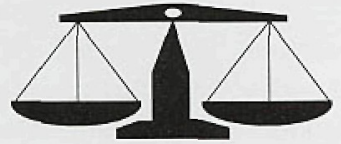

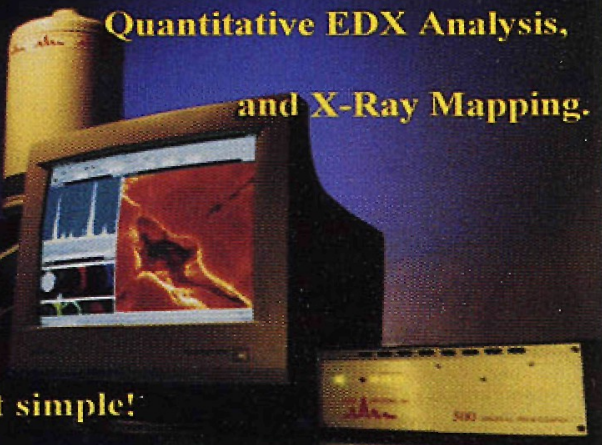

Digital Pulse Processing Electronics High-Resolution Light-Element Si(Li) Detectors High-Resolution Digital Imaging Accurate and Fast Quantitative Analysis Position-Indexed Spectroscopy

Automatic and Customized Particle/Feature Analysis Free Software Updates

Excellent Technical Support

Best Price/Performance Ratio in the Industry

Did we miss something? IXRF $\triangle$ SWSTEMS

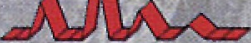

IXRF Systems, Inc.

15715 Brookford Drive

Houston, TX 77059

Tel: (281) 286-6485 Fax: (281) 286-2660

http://www.ixrfsystems.com

\section{STOP \\ HASSLING WITH \\ MULTIPLE \\ SERVICE \\ CONTRACTSE}

START by putting all of your instruments under one service contract with MAS (regardless of make or model). Our expert EM SERVICE GROUP has the knowledge and skill to keep your instrument working at its best.

\section{TEM'S / SEM's}

\section{HITACHI JEOL AMRAY CAMBRIDGE}

WE SERVICE COMPUTER CONTROLLERS BEAM BLANKERS WATER CHILLERS
Contracts and On-Demand Emergency Service at Reasonable Rates from Factory Trained Specialists. 800-421-8451
PREP EQUIPMENT

VACUUM COATERS MECHANICAL PUMPS TURBO PUMPS PLASMA ASHERS SPUTTER COATERS

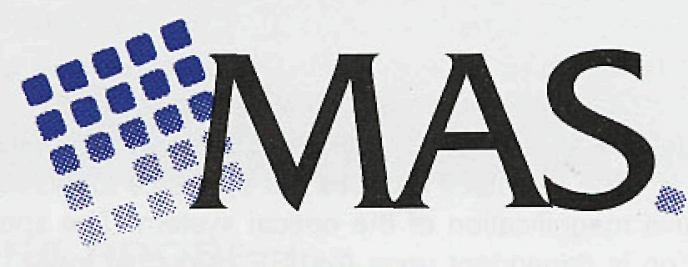

